\title{
Flicker noise comparison of direct conversion mixers using Schottky and HBT dioderings in SiGe:C BiCMOS technology
}

Michaelsen, Rasmus Schandorph; Johansen, Tom Keinicke; Tamborg, Kjeld; Squartecchia, Michele

Published in:

Proceedings of 2015 Integrated Nonlinear Microwave and Millimetre-Wave Circuits Workshop

Link to article, DOI:

10.1109/INMMIC.2015.7330373

Publication date:

2015

Link back to DTU Orbit

Citation (APA):

Michaelsen, R. S., Johansen, T. K., Tamborg, K., \& Squartecchia, M. (2015). Flicker noise comparison of direct conversion mixers using Schottky and HBT dioderings in SiGe:C BiCMOS technology. In Proceedings of 2015 Integrated Nonlinear Microwave and Millimetre-Wave Circuits Workshop (pp. 1-3). IEEE. https://doi.org/10.1109/INMMIC.2015.7330373

\section{General rights}

Copyright and moral rights for the publications made accessible in the public portal are retained by the authors and/or other copyright owners and it is a condition of accessing publications that users recognise and abide by the legal requirements associated with these rights.

- Users may download and print one copy of any publication from the public portal for the purpose of private study or research.

- You may not further distribute the material or use it for any profit-making activity or commercial gain

- You may freely distribute the URL identifying the publication in the public portal 


\title{
Flicker Noise Comparison of Direct Conversion Mixers using Schottky and HBT Dioderings in SiGe:C BiCMOS Technology
}

\author{
Rasmus S. Michaelsen ${ }^{1,2}$, Tom K. Johansen ${ }^{1}$, Kjeld Tamborg ${ }^{2}$, Michele Squartecchia ${ }^{1}$ \\ ${ }^{1}$ Department of Electrical Engineering, Technical University of Denmark, 2800 Kgs. Lyngby, Denmark \\ ${ }^{2}$ Weibel Scientific A/S, 3450 Allerød, Denmark
}

\begin{abstract}
In this paper, we present flicker noise measurements of two X-band direct conversion mixers implemented in a SiGe:C BiCMOS technology. Both mixers use a ring structure with either Schottky diodes or diode-connected HBTs for double balanced operation. The mixers are packaged in a metal casing on an Arlon 25N substrate to shield the sensitive noise measurement. Conversion loss measurements of both mixers is performed both for on-wafer and packaged versions. The experimental results shows that the Schottky diode mixer exhibits a 1/f noise corner frequency of $250 \mathrm{kHz}$, while the diode connected HBT circuit demonstrates a 1/f noise corner frequency around $10 \mathrm{kHz}$.

Index Terms - Mixer, flicker (1/f) noise, MMIC, Schottky diode, diode connected HBT.
\end{abstract}

\section{INTRODUCTION}

Every microwave receiver circuit needs some sort of frequency conversion circuit. Direct conversion has the benefit of giving the modulated signal directly at baseband without the need for complex image rejection structures [1]. For Doppler radars, direct conversion is beneficial as it gives the Doppler shift and thus the speed measurement directly from the received frequency. The key element in a direct conversion receiver is the mixer. The direct conversion mixer has some drawbacks, which comes from the fact that the incoming radio frequency (RF) and local oscillator (LO) frequency is very close. Isolation between LO and RF ports cannot easily be enhanced by using filters so the mixer architecture must have an inherent isolation between these ports. Another drawback is that, due to the low 'intermediate frequency' (IF), it has an increased noise level due to low frequency noise. The low frequency noise is also called flicker noise and is caused by generation-recombination processes in the solid-state mixing devices.

Passive mixers are recognized for their excellent low frequency noise performance. The low frequency noise is influenced by the LO pump signal in a non-linear manner [2] and is generally believed to be present in passive mixers only if an non-zero net average current flows through the mixing core. In double balanced mixers, flicker noise is believed to be caused by unavoidable asymmetries in the diode ring and balun structures. By employing an LO leakage cancelation scheme to the direct conversion mixer, flicker noise can be significantly improved as previously demonstrated by the authors [3].

In general, Schottky diodes is preferred for implementing

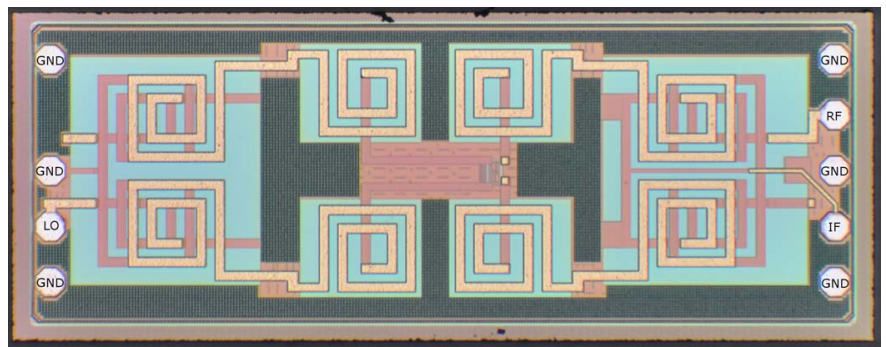

a) Microphotograph of HBT mixer, size $2200 \times 800 \mu \mathrm{m}^{2}$

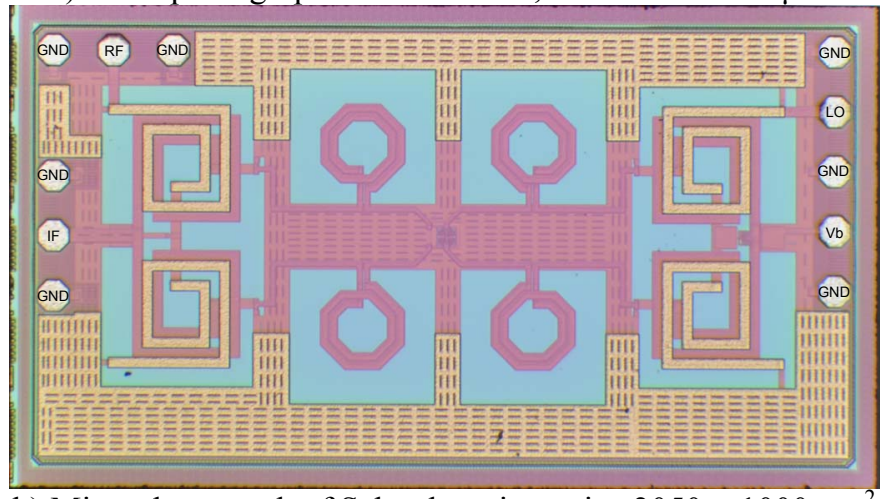

b) Microphotograph of Schottky mixer, size $2050 \times 1000 \mu \mathrm{m}^{2}$ Fig. 1. Microphotograph of the two manufactured chips.

passive mixers because they are majority carrier devices and hence leads to lower conversion loss at higher frequencies than mixers built using pn-junction diodes [4]. Schottky diodes, however, are not standard elements in high performance Silicon technologies. Structures with low reverse leakage current have been proposed in a $\mathrm{SiGe:C}$ BiCMOS technology [5], but little information is generally available about their noise performance at low frequencies. In this paper, we will experimentally investigate the difference in low noise performance between two X-band double balanced direct conversion mixers implemented in SiGe:C BiCMOS technology. One using Schottky diodes, the other using diode connected HBTs.

\section{DOUBled BALANCED MiXer Design}

The double balanced mixers employs lumped element spiral type Marchand baluns on the RF and LO ports due to the good balance achievable over a large bandwidth with this structure. The devices in the diodering are sized to allow easy matching to the Balun structures by placing additional series inductors. 
A thorough description of the mixer circuit using diode connected HBTs is given in [6]. The Schottky diode mixer circuit is similar, but uses a ring of Schottky diodes as the mixer core. A microphotograph of the two manufactured chips is shown in figure 1. Due to the sensitive nature of the flicker noise measurements it is not possible to perform these onwafer. Shielding the mixer from a noisy environment is much easier when packaged in a small metal case. For this purpose the mixer chips is mounted on a Arlon $25 \mathrm{~N}$ substrate with cobber lines covered with soft gold to allow for ball bonding using gold wires. A packaged mixer is shown in figure 2 . Apart from the LO, RF and IF ports also a bulk bias connection to the Silicon substrate is made. This is grounded for the measurements presented here circuits.

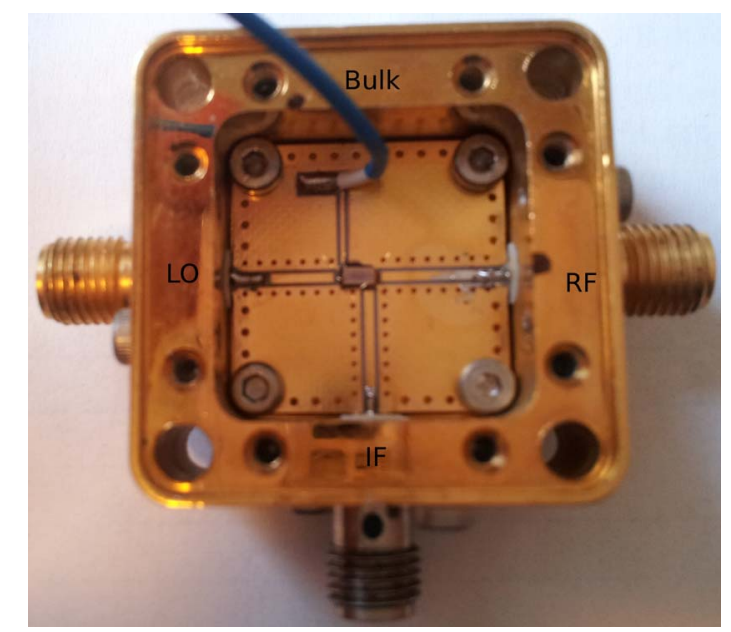

Fig. 2. Packaged mixer chip.

\section{EXPERIMENTAL RESULTS}

In this section the measurement setup will be described. This will be followed by a presentation and discussion of the results of the measurements of flicker noise.

\section{A. Measurement Setup}

The conversion loss is measured, at first, on a bare die using a probe station and then again after packaging. For the onwafer measurements, calibration is performed to a reference plane at the tip of the probe, ie. at the contacts of the chip. For the packaged version, calibration is done to a reference plane at the SMA-connectors. The noise measurement is carried out using a spectrum analyzer together with a calibrated noise source to measure the Y-factor [4]. Two different custom made cavity resonance oscillators with a Q above 10000 are used as LO sources. These deliver an output power around $13 \mathrm{dBm}$ at $9.1 \mathrm{GHz}$ and $10.4 \mathrm{GHz}$, respectively. The noise source is an Anritsu Noise diode with excess noise ratio of 14.3 dB. The IF amplifier is similar to the ones used in commercial Doppler radars manufactured by Weibel Scientific and has a gain of $80 \mathrm{~dB}$. The measurement setup is shown in figure 3 . As the present investigation is concerned with the comparison between the two mixer circuits, there has not been done extensive measurements to determine the noisecontributions from the oscillator and IF-amplifier, although quality components has been used to minimize the effect.

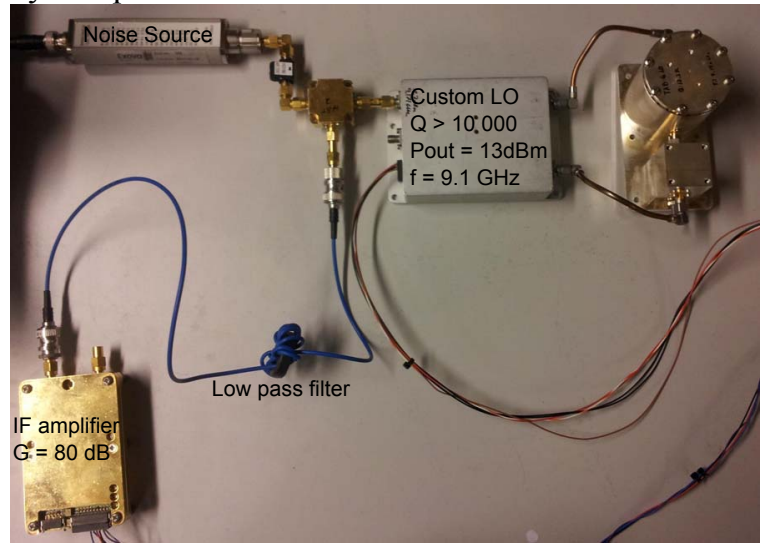

Fig. 3. Measurement setup for noise characterization.

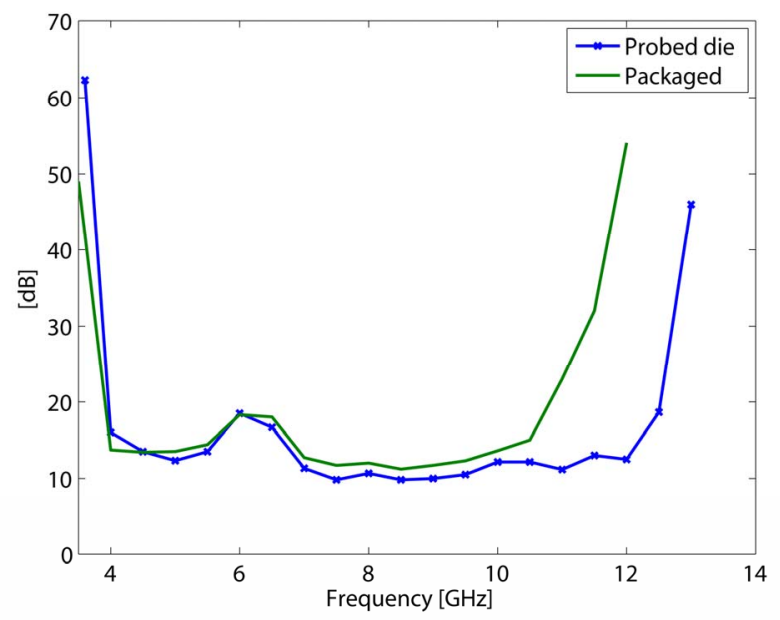

Fig. 4. Measurement of diode-connected HBT mixer conversion gain versus frequency, for probe measured die and packaged die.

\section{B. Measurements Results and Discussion}

In this subsection, the measurements results are discussed. The conversion loss as a function of RF frequency for the diode connected HBT mixer is shown in figure 4 both for the probed and packaged version. The conversion loss is lowest at $8.5 \mathrm{GHz}$ where it is $9.8 \mathrm{~dB}$ for the probed and $11.2 \mathrm{~dB}$ for the packaged version. Around $9 \mathrm{GHz}$ where the noise is measured the conversion loss is $10 \mathrm{~dB}$ for the probed and $11.7 \mathrm{~dB}$ for the packaged version. For the Schottky diode mixer the conversion loss is plotted as a function of frequency in figure 5. The conversion loss is lowest at $11 \mathrm{GHz}$ where it is $8.8 \mathrm{~dB}$ for the probed and $11.2 \mathrm{~dB}$ for the packaged version. At 10.4 $\mathrm{GHz}$ where the noise is measured the conversion loss is $9.3 \mathrm{~dB}$ for the probed and $11.4 \mathrm{~dB}$ for the packaged version. The additional loss of roughly $\sim 1.5 \mathrm{~dB}$, which was observed for both mixers, is seen across the entire bandwidth, and is what could be expected from the bonding wires, microstrip lines 
and SMA to microstrip connections. Apart from additional loss, the bandwidth is also reduced by the added inductance on the input terminals.

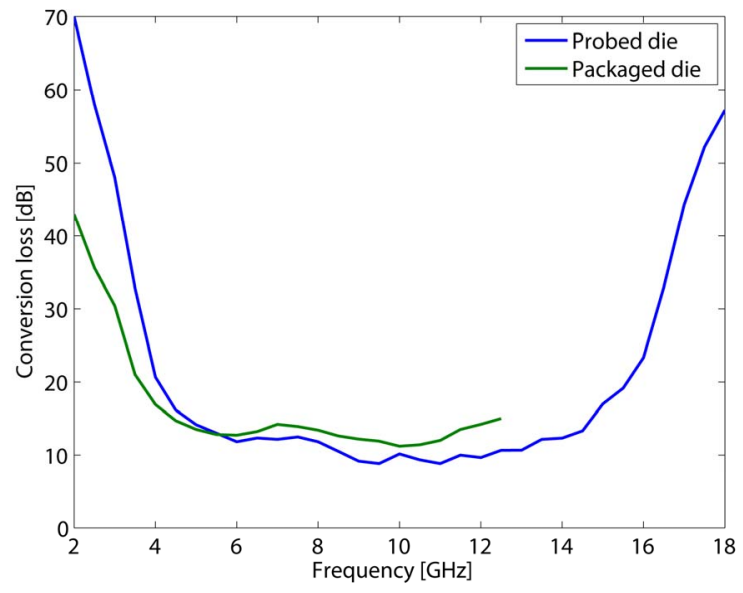

Fig. 5. Measurement of Schottky mixer conversion gain versus frequency, for probe measured die and packaged die.

The noise figure of the Schottky diode mixer is plotted in figure 6 , for IF frequencies from $50 \mathrm{kHz}$ to $1 \mathrm{MHz}$, with a LO frequency of $10.4 \mathrm{GHz}$. At higher frequencies the noise figure is $9.0 \mathrm{~dB}$ and rises as $1 / \mathrm{f}$ with a corner around $250 \mathrm{kHz}$, with a noise figure around $22 \mathrm{~dB}$ at $50 \mathrm{kHz}$. In comparison, the diode-connected HBT mixer demonstrates a noise figure of $8.5 \mathrm{~dB}$ with a LO frequency of $9.1 \mathrm{GHz}$. Both mixers operate with a 3-dB bandwidth covering the entire X-band, but the Schottky diode mixer operates best around $10 \mathrm{GHz}$ and the diode-connected HBT mixer around $8.5 \mathrm{GHz}$. To give a realistic view of the diode-connected HBT mixer, the noise figure with a LO frequency of $9.1 \mathrm{GHz}$ is plotted in figure 7 on a logartihmic scale from 1-100 KHz. Here it is seen that the diode-connected HBT mixer also have a $1 / \mathrm{f}$ tendency, but with a corner frequency as low as $10 \mathrm{kHz}$. Now it is clearly demonstrated that the HBT-connected diodes perform much better than the Schottky diodes in terms of low frequency noise performance. The higher low frequency noise in the Schottky diode mixer is believed to be caused by poor quality of the Schottky barrier and effect of leakage current.

\section{CONCLUSION}

We have presented measurement of low-frequency noise of two mixers in $\mathrm{SiGe} \mathrm{C}$ BiCMOS technology, one utilizing Schottky diodes the other diode connected HBTs. To perform the measurements the mixers has been mounted on an Arlon $25 \mathrm{~N}$ substrate, gold ball bonded to soft gold transmission lines, and enclosed in a metal casing, thus shielding them from external noise sources. The noise measurements are performed using the $\mathrm{Y}$-factor method. The experimental results showed that the Schottky diode mixer exhibits a higher $1 / \mathrm{f}$ noise with a corner frequency around $250 \mathrm{kHz}$, while the diode connected HBT circuit has a 1/f noise corner frequency around $\sim 10 \mathrm{kHz}$.

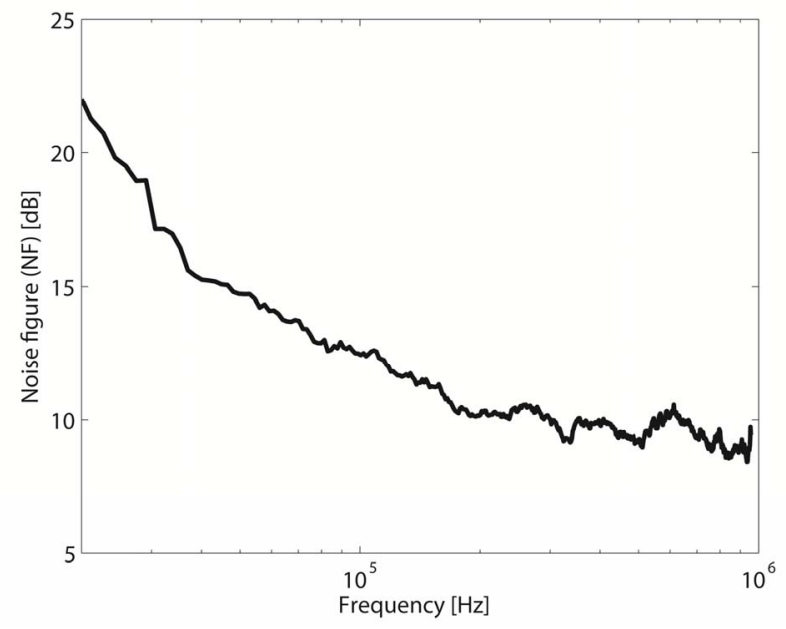

Fig. 6. Measurement of Schottky mixer noise figure as a function of IF frequency.

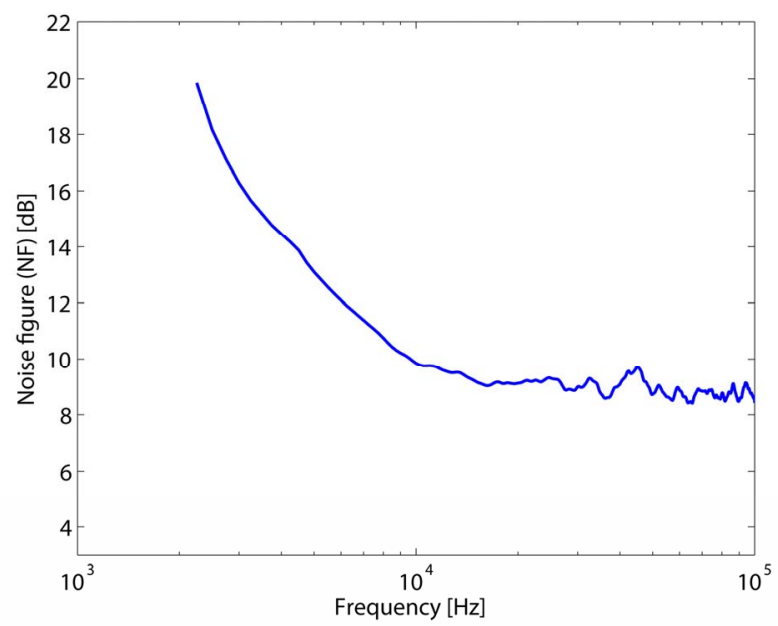

Fig. 7. Measurement of HBT mixer noise figure as a function of IF frequency.

\section{REFERENCES}

[1] B. Razavi, "Design consideration for direct-conversion receivers," IEEE Trans. Circuits and Systems II: Analog and Digital Signal Processing, vol. 44, no. 6, pp. 428-435, 1997.

[2] M. Rudolph and F. Bonani, "Low-Frequency Noise in Nonlinear Systems", IEEE Microwave Magazine, pp. 84-92, Feb. 2009.

[3] R. S. Michaelsen, T. K. Johansen, and K. Tamborg, "Investigation of LO-leakage cancellation and DC-offset influence on flicker-noise in X-band mixers," in 7th European Microwave Integrated Circuit Conference, 2012, pp. 99-102.

[4] S. A. Maas, Microwave Mixers, 2nd ed. Artech House, 1993.

[5] R. M. Rassel et al., "Schottky Barrier Diodes for Millimeter Wave SiGe BiCMOS Applications," in IEEE BCTM, 2006. 2014.

[6] R. S. Michaelsen, T. K. Johansen, K. Tamborg, and V. Zhurbenko, "Design of a broadband X-band double-balanced mixer in SiGe HBT technology," Int. Jour. Microwave and Wireless Tech., vol. 6, no. 3-4, pp. 235.242, 2014. 